\title{
A systematic review and meta-analysis of the effect of an ankle-foot orthosis on gait biomechanics after stroke
}

Clinical Rehabilitation

27(10) 879-891

(C) The Author(s) 2013

Reprints and permissions:

sagepub.co.uk/journalsPermissions.nav

DOI: 10.1 177/02692/55।3486497

cre.sagepub.com

@SAGE

\author{
SF Tyson 1,2, E Sadeghi-Demneh ${ }^{2,3}$ and CJ Nester ${ }^{2}$
}

\begin{abstract}
Objective: To systematically review the evidence on the effects of an ankle-foot orthosis on gait biomechanics after stroke

Data sources: The following databases were searched; AMED, CINHAL, Cochrane Library (Stroke section), Medline, PubMed, Science Direct and Scopus. Previous reviews, reference lists and citation tracking of the selected articles were screened and the authors of selected trials contacted for any further unpublished data.

Review methods: Controlled trials of an ankle-foot orthosis on gait biomechanics in stroke survivors were identified. A modified PEDro score evaluated trial quality; those scoring 4/8 or more were selected. Information on the trial design, population, intervention, outcomes, and mean and standard deviation values for the treatment and control groups were extracted. Continuous outcomes were pooled according to their mean difference and $95 \%$ confidence intervals in a fixed-effect model.

Results: Twenty trials involving 3/4 participants were selected. An ankle-foot orthosis had a positive effect on ankle kinematics $(P<0.0000 \mathrm{I}-0.0002)$; knee kinematics in stance phase $(P<0.000 \mathrm{I}-0.0 \mathrm{I})$; kinetics $(P=0.000 \mathrm{I})$ and energy cost $(P=0.004)$, but not on knee kinematics in swing phase $(P=$ 0.84), hip kinematics $(P<0.18-0.89)$ or energy expenditure $(P=0.43)$. There were insufficient data for pooled analysis of individual joint moments, muscle activity or spasticity. All trials, except one, evaluated immediate effects only.

Conclusions: An ankle-foot orthosis can improve the ankle and knee kinematics, kinetics and energy cost of walking in stroke survivors.
\end{abstract}

\section{Keywords}

Foot and ankle, gait analysis, biomechanics, orthoses, stroke

Received: 28 January 20I3; accepted: 22 March 2013

'Stroke and Vascular Research Centre and School of Nursing, Midwifery and Social Work, University of Manchester, Manchester, UK

${ }^{2}$ School of Health Sciences, University of Salford, Salford, UK ${ }^{3}$ Musculoskeletal Research Centre, Isfahan University of Medical Sciences, Isfahan, Iran

\section{Corresponding author:}

Sarah Tyson, Stroke and Vascular Research Centre and School of Nursing, Midwifery and Social Work, University of Manchester, Jean McFarlane Building, Oxford Road, Manchester MI3 9PL, UK.

Email: Sarah.Tyson@manchester.ac.uk 


\section{Introduction}

Regaining independent safe mobility is a frequent goal of stroke rehabilitation ${ }^{1}$ and an ankle-foot orthosis is often used to improve balance and mobility as a part of such a programme. In a recent systemic review focusing on the impact of an ankle-foot orthosis on function, the authors of this paper $^{2}$ demonstrated that an ankle-foot orthosis can improve walking impairments, walking activity and balance in people with stroke. However, the review did not address the effect of an ankle-foot orthosis on gait biomechanics. Yet, this is an important element of the evidence base as biomechanics relate to the mechanism of action. An understanding of the mechanisms of action is important for accurate prescription of the most appropriate design of ankle-foot orthosis for an individual patient and to develop more effective designs.

The only other systemic review ${ }^{3}$ of the effects of an ankle-foot orthosis also reported a beneficial effect on function and the temporo-spatial aspects of gait; however it was completed over 10 years ago and did not include pooled meta-analysis. Thus our aim was to systematically review the evidence for the impact of an ankle-foot orthosis on gait biomechanics (in terms of kinetics, kinematics, muscle activity and energy expenditure) in people with stroke using contemporary searches and pooled meta-analysis where possible. The effect of an ankle-foot orthosis on the temporospatial parameters of gait have been reported previously. $^{2}$

\section{Methods}

The following databases were searched from inception to November 2011; AMED, CINAHL, the Stroke section of the Cochrane Library, OVIDMedline, PubMed, Science Direct and Scopus. In addition, previous literature reviews on ankle-foot orthoses for people with stroke, reference lists and citation tracking of the selected articles were screened. The authors of selected trials were contacted to ask whether they had any further unpublished data. The search strategy included a combination of three groups of keywords as follows:

- condition-related: 'stroke', 'hemiplegi*', 'cerebrovascular accident'

- intervention-related: 'ankle foot orthos*', 'AFO', 'orthotic', 'brace', 'leaf-spring', and 'calliper'

- outcome-related: 'biomechanic*', 'kinematic*', 'kinetic*', 'muscle activity', 'EMG', 'energy', and 'oxygen consumption'.

\section{Selection criteria}

Controlled trials (including cross-over or paired sample designs) published in English, which involved adult stroke survivors and assessed the effects of an ankle-foot orthosis on biomechanical aspects of hemiplegic gait (kinematics, kinetics, muscle activity or energy consumption) compared to walking with no ankle-foot orthosis (with shoes or barefeet) were selected. Studies that included people with other conditions were included if at least $50 \%$ of the participants were stroke survivors or the data for the stroke survivors could be extracted. Uncontrolled trials, case reports and single-case designs were excluded due to the high risk of bias in these designs. The titles, abstracts and then full text of the papers identified by the search were screened by two independent reviewers (the authors, ESD and SFT) to identify those that met the selection criteria and extract the data. Decisions about which trials to select were made by negotiation. A third party was available to arbitrate but was not needed.

\section{Methodological quality assessment}

The methodological quality of the trials which met the selection criteria was then assessed using a modified PEDro scale (detailed in Table 1 online). The PEDro scale 4 is a widely used checklist of 11 criteria to assess the risk of bias and thoroughness of reporting in trials. For this review, some criteria were amended to address issues relevant to designs used in orthotic research. The criterion of 'blinding 
the therapist' was deleted as it is not possible to blind either healthcare professional or patient to whether they are wearing an ankle-foot orthosis or not. Equally, it is not possible to blind the assessor to whether an ankle-foot orthosis is worn or not ('assessor blinding'), but it is possible to minimize the bias by using an outcome measure which does not require any (or minimal) judgement, such as an automated measurement system. Therefore the criterion to assess whether a 'blinded assessor' was used was changed so that a 'pass' was obtained if an automated measurement system was used rather than the assessor being blinded. When scoring the modified PEDro scale, cross-over designs were given positive score for 'blind allocation' if all participants received all conditions because the assessor cannot influence group allocation when the subjects receive all treatments. These modifications produced a checklist of eight items with a maximum score of 8 .

The quality of the evidence from the selected trials was then arbitrarily classified into three levels. Articles that scored $7-8 / 8$ on the modified PEDro scale were rated as good methodological quality. Those that scored 4-6 were rated as moderate quality, and those scoring 0-3 were classified as poor quality. Only the good and moderate quality studies were selected for analysis.

\section{Data extraction}

Information on the trial design, population recruited, intervention delivered, outcomes measured and the mean and standard deviation values for the treatment and control groups were independently extracted by the authors (SFT and EDS) from the selected trials (Table 2).

\section{Statistical analysis}

Where possible, continuous outcomes were pooled according to their weighted mean difference (WMD) and $95 \%$ confidence intervals in a fixedeffect model using 'Review Manager' software (RevMan 5). Where trials had used different parameters to measure of the same underlying construct a standardized mean difference (SMD) and
95\% confidence intervals with a fixed-effect model was calculated. If statistical heterogeneity exceeded $50 \%$, a random effect model was used. ${ }^{5}$ We attempted to use general inverse variance to analyse cross-over studies but insufficient studies reported their data in a format that could be used for this analysis. Consequently cross-over studies were analysed as if they had used a parallel-group design using the mean difference or standardized mean difference as appropriate, although we recognized that this was likely to give a conservative estimate of the effect. ${ }^{6}$ However, this over-counts the number of participants because the default settings in the RevMan software assume that the control and treatment groups are different. In the resulting Forrest plots of the meta-analyses, the true numbers of participants are added as a footnote. Where pooling of data was not possible, a narrative analysis was undertaken. Parameters for which only one trial was identified are not reported as no analysis was possible.

\section{Results}

Initially 1110 titles were identified; 180 abstracts screened and 65 full texts obtained. Of these 28 met all selection criteria and were chosen for the quality assessment and 23 were of high or moderate quality. ${ }^{7-29}$ Four were later rejected from the pooled analysis, ${ }^{8,9,11,20}$ as they did not provided data on the variability of the reported outcomes or data could not be extracted from graphical presentations and the data could not be obtained by contacting the authors. We obtained data to enable pooled analysis from the authors of four trials. ${ }^{14,17-19}$ Thus 20 trials were selected for the analysis, the details of which are shown in Table 2.

The selected trials involved 314 participants in small sample sizes, ranging from 5 to 32 participants; none reported a sample size calculation. A non-randomized controlled cross-over trial (or comparison with : without ankle-foot orthosis) was the most frequent design (10/20 trials $)^{15-19,22-24,26,28}$ in which walking without an ankle-foot orthosis was the control condition but the order of testing was not randomized. A randomized cross-over 


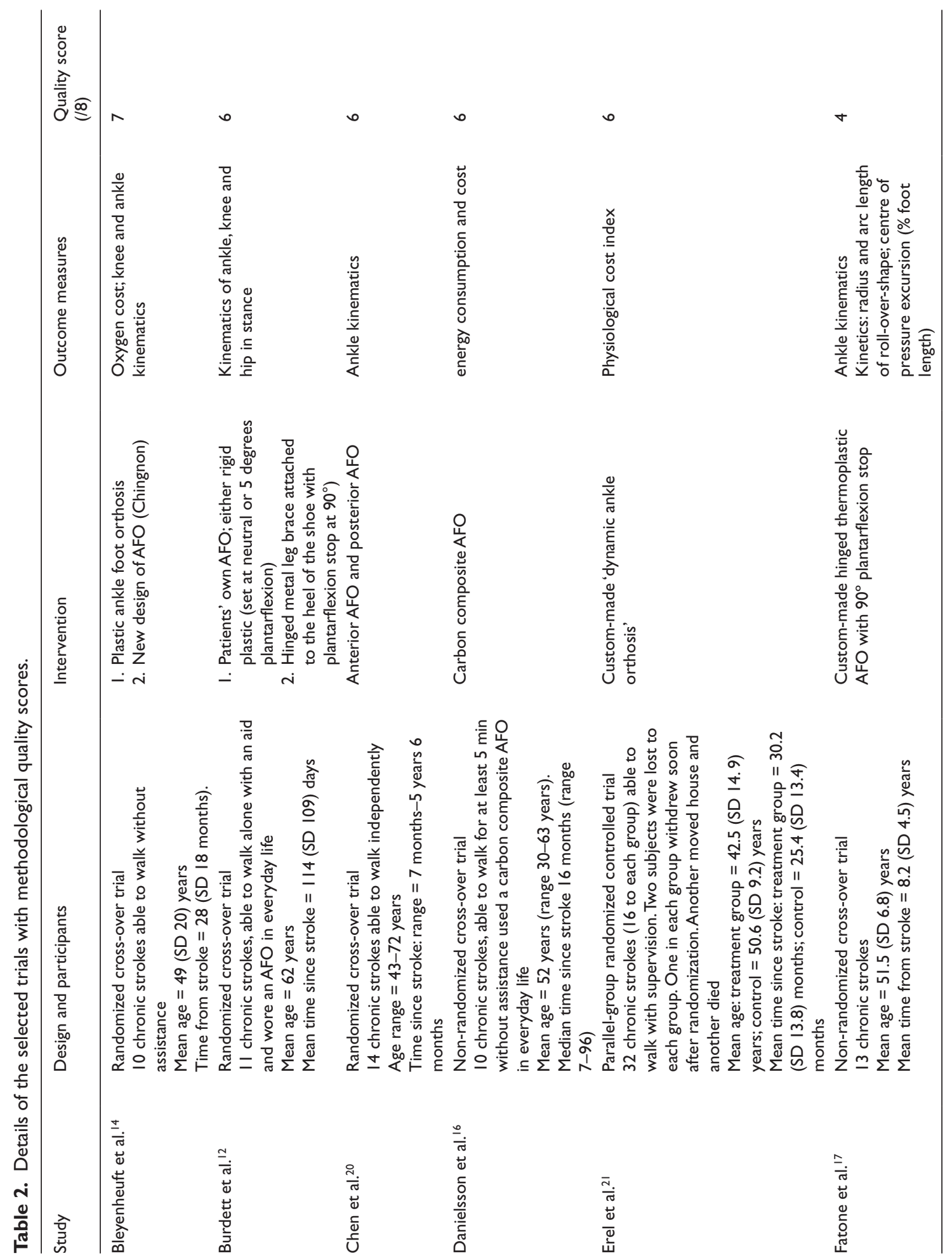




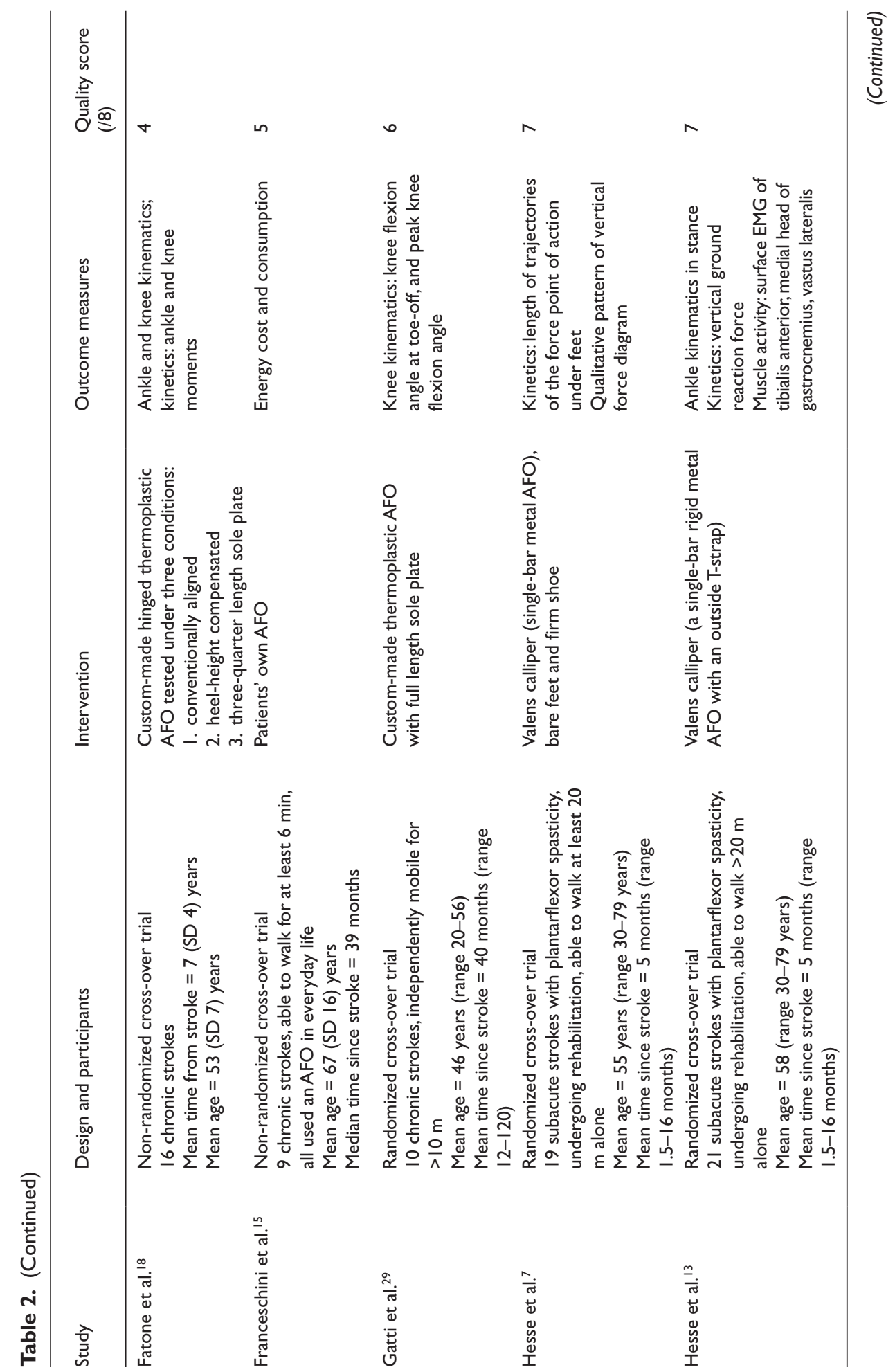




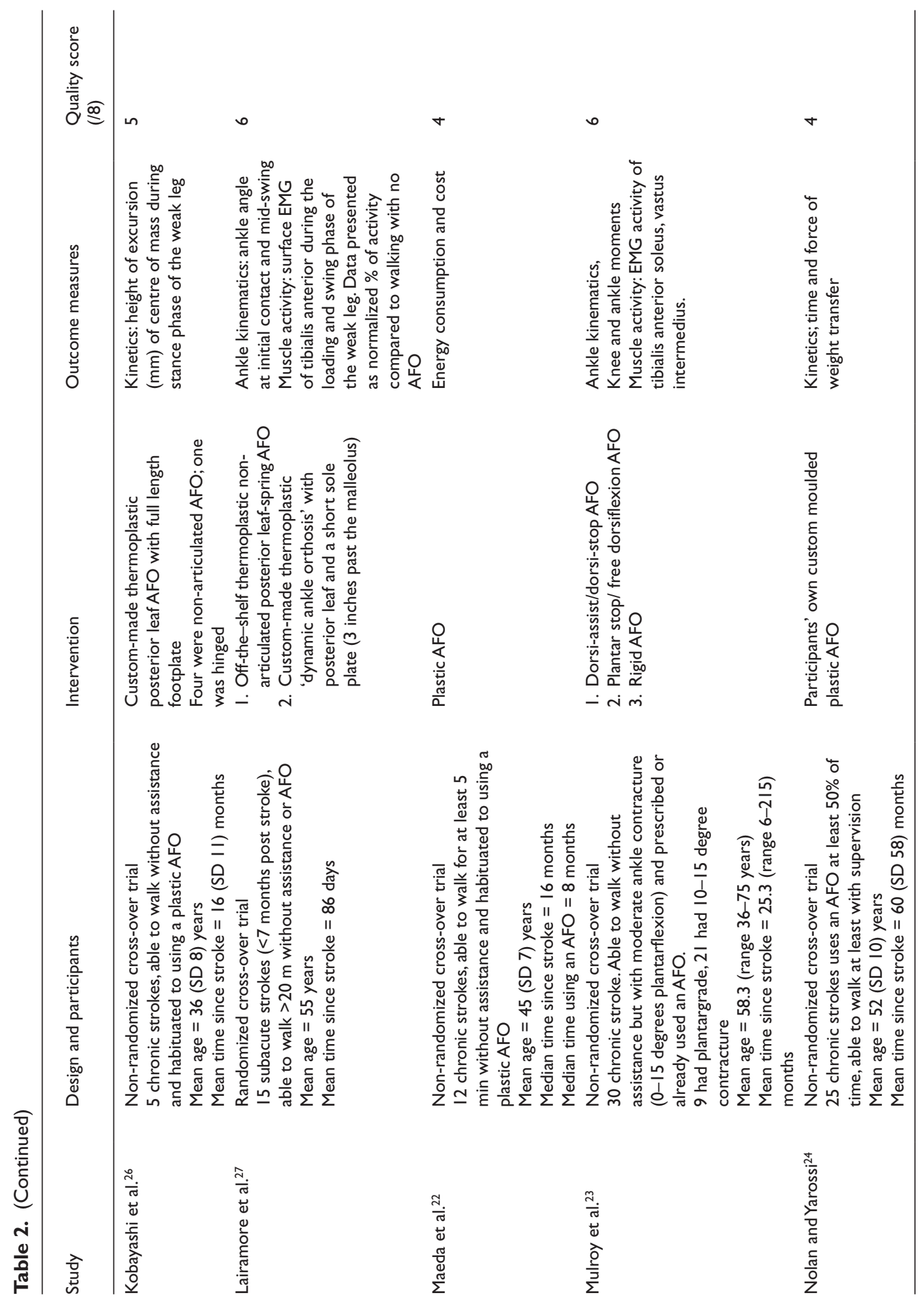




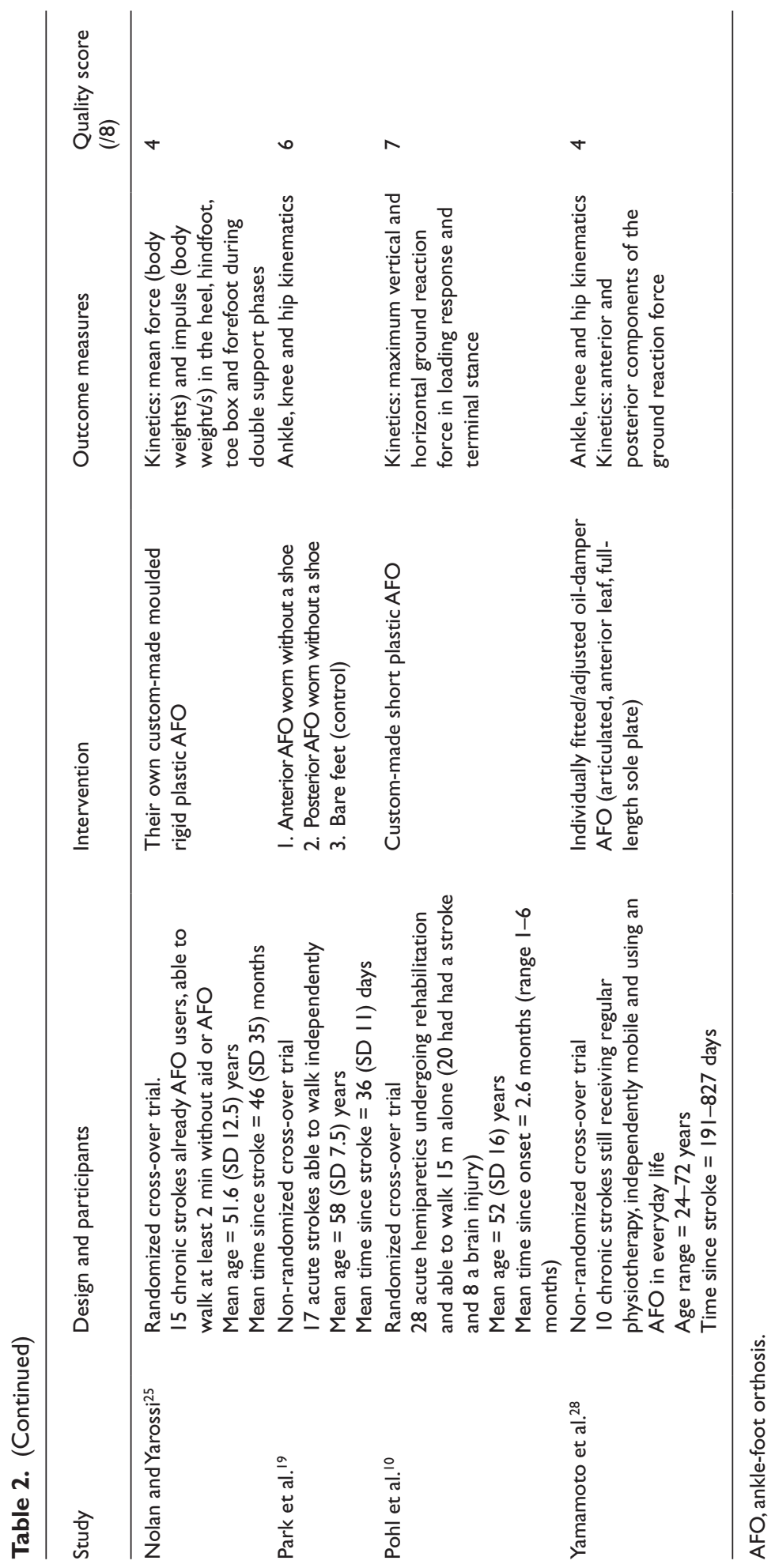


trial was used in nine trials $7,10,12-14,20,27,29$ in which walking without an ankle-foot orthosis was the control condition; the randomization came from the order of testing condition. One trial used a parallel-group randomized controlled design ${ }^{21}$ in which one group of participants were treated with an ankle-foot orthosis and the control group wore shoes only. Both groups were tested after three months of daily wear.

The quality of selected trials are detailed in Table 2; six trials $7,10,13,14,27,29$ were of good quality while the others were moderate. None reported the effect size of interventions (between-group statistics). The participants were usually convenience samples typically recruited from patients known to a clinical service such as physiotherapy, orthotics or gait assessment. Inclusion criteria were generally broad and included a wide spectrum of age, time since stroke and sensorimotor levels. All participants had previously used an ankle-foot orthosis either in everyday life or for a short time before the testing session (at least one week). In most studies, independent walking (without aids or another person) was the minimum inclusion criterion; except four studies in which the assistive devices were acc epted. ${ }^{12,15,16,22,27,28}$ Some studies had additional minimum criteria relating to walking ability, such the time ${ }^{15,18}$ or distance ${ }^{7,13,19,27,29}$ participants could walk. In addition, Hesse and co-workers ${ }^{7,13}$ specifically sought participants with marked spasticity but no ankle contracture, while Mulroy et al. recruited participants with moderate ankle contracture ${ }^{23}$ and Erel et al. specified that they recruited participants with no spasticity or contracture. ${ }^{21}$ Most trials specified that participants should be in the chronic stages of stroke ( $>6$ months $)^{14,15,18,21-23,26,29}$ but others involved participants in the acute ( $<3$ months) and subacute (3-6 months) stages. ${ }^{7,12,13,27}$

\section{Analysis of effect of an ankle-foot orthosis}

The results are described according to the classification of biomechanical outcomes: kinematics, kinetics, muscle activity and energy expenditure. The $P$-values of the comparisons are presented in the text. Further details of the mean differences,
$95 \%$ confidence intervals and effect sizes are shown in Table 3. Figures 1-5 (online) show the Forrest plots of the pooled analyses.

\section{Kinematics}

- Ankle kinematics: Seven studies ${ }^{12,14,17,18,23,27,28}$ involving 106 participants demonstrated an increase in dorsiflexion at initial foot contact/ heel strike when using an ankle-foot orthosis $(P<0.0001)$. Seven studies ${ }^{12,14,17-19,23,28}$ of 95 participants showed an increase in peak ankle dorsiflexion during stance with an ankle-foot orthosis $(P<0.0002)$. Eight studies ${ }^{12,14,17-19,23,27,29}$ involving 122 participants found an increase in peak dorsiflexion in swing phase $(P<0.00001)$ and two studies ${ }^{12,23}$ of 41 participants found increased peak dorsiflexion at toeoff $(P<0.00001)$ with an ankle-foot orthosis.

- Knee kinematics: Four studies ${ }^{12,14,23,28}$ of 61 subjects found an increase in knee flexion at initial contact $(P<0.02)$ with an ankle-foot orthosis, while five studies ${ }^{14,16,21,25,30}$ of 78 participants demonstrated an increase in peak knee flexion at loading response with an ankle-foot orthosis $(P<0.007)$. Five studies ${ }^{14,18,19,23,28}$ (83 participants) showed improved peak knee extension in stance phase with the ankle-foot orthosis $(P<0.01)$ but no effect on peak knee flexion in swing phase $(P<0.72)(n=93)$ with an anklefoot orthosis.

- Hip kinematics: Three studies ${ }^{12,19,28}$ involving 46 participants evaluated the impact of an ankle-foot orthosis on hip kinematics. Two common parameters could be extracted, both involving only two studies. Two studies ${ }^{12,28}$ $(n=21)$ evaluated the effect of an ankle-foot orthosis on peak hip flexion at initial contact/ heel strike and found no effect $(P<0.89)$, which was reiterated when the effect of an ankle-foot orthosis on peak hip extension during stance phase was examined ${ }^{19,28}$ in 27 patients $(P<0.18)$.

Kinetics. Two studies ${ }^{7,18}$ involving 35 participants showed an increase in the length of centre of pressure excursion under the affected foot during stance 
Table 3. The results of pooled-data analysis; the number of studies and participants, mean differences (including $95 \%$ confidence intervals) and effects size.

\begin{tabular}{|c|c|c|c|c|}
\hline Pooled outcomes & $\begin{array}{l}\text { Number of } \\
\text { studies }\end{array}$ & Subjects & Mean difference $(95 \% \mathrm{Cl})$ & $P$-value \\
\hline \multicolumn{5}{|l|}{ Kinematics (degrees) } \\
\hline \multicolumn{5}{|l|}{ Ankle } \\
\hline $\begin{array}{l}\text { Ankle angle at initial contact } \\
\text { (degrees) }\end{array}$ & 7 & 106 & $8.58(7.55,9.60)$ & $0.00001 *$ \\
\hline $\begin{array}{l}\text { Peak dorsiflexion during stance } \\
\text { phase (degrees) }\end{array}$ & 7 & 95 & $2.15(1.04-3.26)$ & $0.0002^{*}$ \\
\hline $\begin{array}{l}\text { Peak dorsiflexion during swing } \\
\text { phase (degrees) }\end{array}$ & 8 & 122 & $6.62(5.43,7.820)$ & $0.00001 *$ \\
\hline $\begin{array}{l}\text { Peak dorsiflexion at toe-off } \\
\text { (degrees) }\end{array}$ & 2 & 41 & $5.01(3.04,6.99)$ & $0.000^{*}$ \\
\hline \multicolumn{5}{|l|}{ Knees } \\
\hline $\begin{array}{l}\text { Knee flexion at initial contact } \\
\text { (degrees) }\end{array}$ & 4 & 61 & $2.40(0.20,4.61)$ & $0.02 *$ \\
\hline $\begin{array}{l}\text { Peak knee flexion at loading } \\
\text { response (degrees) }\end{array}$ & 5 & 78 & $3.11(0.85,5.36)$ & $0.007^{*}$ \\
\hline $\begin{array}{l}\text { Peak knee extension during } \\
\text { stance phase (degrees) }\end{array}$ & 5 & 83 & $2.69(0.64,4.78)$ & $0.0 I^{*}$ \\
\hline $\begin{array}{l}\text { Peak knee flexion during swing } \\
\text { phase (degrees) }\end{array}$ & 6 & 93 & $0.48(-2.18,3.15)$ & 0.72 \\
\hline \multicolumn{5}{|l|}{ Hip } \\
\hline $\begin{array}{l}\text { Peak hip flexion at initial contact } \\
\text { (degrees) }\end{array}$ & 2 & 21 & $0.25(-3.49,4.10)$ & 0.89 \\
\hline $\begin{array}{l}\text { Peak hip extension during stance } \\
\text { phase (degrees) }\end{array}$ & 2 & 27 & I.8I $(0.83,4.45)$ & 0.18 \\
\hline \multicolumn{5}{|l|}{ Kinetics } \\
\hline $\begin{array}{l}\text { COP excursion under foot (\% of } \\
\text { foot length) }\end{array}$ & 2 & 35 & $25.70(20.47,30.94)$ & $0.0001 *$ \\
\hline \multicolumn{5}{|l|}{ Energy } \\
\hline $\begin{array}{l}\text { Metabolic energy cost }(\mathrm{mL} \mathrm{O} / \\
\mathrm{kg} / \mathrm{m})\end{array}$ & 3 & 37 & $-0.70(-1.18,-0.23)$ & $0.004 *$ \\
\hline $\begin{array}{l}\text { Oxygen consumption }(\mathrm{mL} \mathrm{O} / \mathrm{kg} / \\
\mathrm{min})\end{array}$ & 3 & 37 & $-0.19(-0.64,0.27)$ & 0.43 \\
\hline
\end{tabular}

*Statistically significant difference.

with the ankle-foot orthosis $(P<0.0001)$. Six other trials ${ }^{13,14,17,23,26,28}$ involving 99 participants measured aspects of the kinetics but there were no common parameters that could be pooled. All reported a significantly positive effect with an ankle-foot orthosis except Yamamoto et al. ${ }^{28}$ who had reported mixed results in only 10 patients.

Energy expenditure. Three studies ${ }^{15,18,22}$ involving 31 participants evaluated energy expenditure and cost and found an improvement in energy cost $(P<$ $0.004)$ but no effect on energy consumption $(P<$ 0.43 ) with an ankle-foot orthosis. For this calculation, a standardized mean difference was calculated as the data were collected for different time periods (6 minutes ${ }^{15,22}$ or 5 minutes $\left.^{16}\right)$. In addition, Erel et al. ${ }^{21}$ who used a parallel-group randomized controlled trial design found that an ankle-foot orthosis had a beneficial effect on the Physiological Cost Index $(P<0.001$ with a large effect size (1.61). 
Muscle activity. Three trials measured muscle activity ${ }^{13,23,27}$ in 66 patients but used incompatible parameters so there were insufficient data for a pooled analysis. None of the selected trials measured spasticity parameters.

Statistical heterogeneity. Statistical heterogeneity requiring a random effects model was not found in any of the analyses.

\section{Discussion}

The results of this systematic review suggest that an ankle-foot orthosis can have a beneficial effect on knee and ankle kinematics by:

- preventing foot-drop (i.e. plantarflexion) in early stance, swing phase and toe-off;

- facilitating weight-bearing on the paretic leg by increasing the excursion of the centre of pressure forwards over the stance foot, enhancing knee movements during stance phase;

- reducing the energy cost of walking.

We found no effect on hip kinematics (but these analyses were probably under-powered). There were insufficient data to analyse the effect on muscle activity, spasticity or ankle, hip and knee kinetics.

The only previous review of this topic ${ }^{3}$ was limited to a narrative analysis and included trials selected in this review. Not surprisingly they also found that an ankle-foot orthosis had a positive effect on ankle kinematics and energy expenditure and insufficient data to draw a conclusion about the effect on muscle activity. Our companion paper, which shares many of the same trials and addresses function and temporo-spatial parameters of gait also found a positive effect in terms of walking impairment, activity and balance. ${ }^{2}$

The selected trials in this review were predominantly cross-over trials assessing immediate effects in small, highly selected samples. A cross-over trial is an effective design to measure immediate effects because the control and intervention groups are the same people thereby reducing heterogeneity and minimising the required sample size. Testing sessions were completed in a single day. This is an effective way to evaluate the biomechanics, with minimal drop-out rates, but it means that the effects of long-term use remain largely unexamined. It is unknown whether an ankle-foot orthosis continues to impact on the patients' gait pattern in the long term, or whether the patient adapts to the ankle-foot orthosis and returns to their previous pattern. Alternatively, long-term use could facilitate motor learning that would enable the patient to walk with an improved gait pattern once the ankle-foot orthosis is removed. Further parallel-group randomized controlled trials of the short- and long-term effects in people with chronic stroke and those undergoing rehabilitation are needed to test these hypotheses. The feasibility of such trials has been demonstrated recently. ${ }^{21}$

Similarly, it is not known whether any changes continue once the ankle-foot orthosis is taken off. The research design used in the selected trials assume that this does not happen with short-term use and there are no carry-over effects between testing conditions; our findings support this view as we found significant changes in gait pattern (and in function in our companion review ${ }^{2}$ ) immediately when the patient was or was not wearing an anklefoot orthosis.

Only half the selected trials used a randomized design. Consequently the risk of bias in the analysis is moderately high and the results need to be treated with more caution than would be necessary if all of the trials had been randomized. Randomization of this type of cross-over trial is a simple matter requiring no additional resources or time; it merely affects the order of testing. Given the simplicity to randomize and the inherent bias in an unrandomized design, their continued use is difficult to justify. Future trials should include a randomized design as a priority.

A further design feature that limits the strength of the conclusions which can be drawn is the small, highly selected sample sizes. None of the selected trials used a sample size calculation or gave an explanation for the numbers recruited and the 
sample sizes were small. Even when data were pooled, none of the analyses included more than 122 participants and so may have been underpowered. Furthermore the samples recruited were selected, by and large, from patients known to a service or the researchers. As such they could be considered highly convenient; future trials need to recruit pragmatic samples to enhance generalizability.

Although the results show that highly statistically significant differences were found, the clinical or functional significance of the differences are unclear. For several parameters, the mean differences were actually very small (a matter of a few degrees of movement) and it is not known whether such changes are sufficient to produce a meaningful and important difference to the patient in terms of function or comfort. However our companion paper, which includes many of the same trials, found improvements in walking speed and stride or step length impairment which suggests that the changes are sufficient to translate into function. ${ }^{2}$ Future trials need to include measures of walking activity as well as biomechanics to explore this relationship further. They also need to include sample sizes with sufficient power to detect a clinically and functionally meaningful effect (if it exists). Other important outcomes such as the impact an ankle-foot orthosis on falls and patients' confidence also need to be included.

Like any review, the strength of our conclusions is dependent on the completeness of the data identified. We were only able to include publications in English and so we may have missed publications in other languages. However, as well as database searches we contacted authors and original researchers for further data, information about the selected trials' design and checking the reported data; therefore the risk of publication bias is expected to be low.

During the analysis we included 'walking without an ankle-foot orthosis' as the control condition. This included trials in which participants walked in shoes and others in which they were barefoot. Trials in which walking barefoot was the control were mainly from eastern countries where it is uncommon to wear shoes indoors. As no differences in temporospatial and balance parameters when walking barefoot compared with wearing shoes have been reported ${ }^{7}$ we felt justified in combining the two control groups.

Despite these limitations we were able to pool data; primarily kinematic and concerning the ankle. One of our aims for this review was to explore an ankle-foot orthosis' mechanism of action, which has been achieved. An ankle-foot orthosis is traditionally used to prevent foot-drop (excessive plantarflexion) during swing phase and promote heel strike in early stance. Our findings confirm that an ankle-foot orthosis has this effect. But we also found that it can impact on the biomechanics of stance phase, particularly knee extension, dorsiflexion and weight transference over the stance foot, in that the ankle-foot orthosis can prevent excessive plantarflexion and knee extension during the loading phase of stance and 'steer' the advancing body weight over the foot. As the stance foot is fixed on the floor, this must occur by avoiding posterior transition of the tibia over the fixed foot, which allows dorsiflexion and more normal knee movements (greater knee flexion in early-mid stance and greater knee extension in late stance and at push-off) as the body weight is transferred over the foot. This would apply a stretching force on calf muscles, thereby positioning the muscle fibres in a more efficient length before the onset of muscle contraction, ${ }^{30}$ which would, in turn, explain the reduction in energy cost as simultaneous plantarflexion, knee extension and hip extension at push-off is thought to bring the centre of gravity higher and make the gait pattern more efficient. ${ }^{30,31}$ It would also explain how excursion of the centre of pressure improved with an ankle-foot orthosis (indicating a more symmetric, balanced gait) $7,17,26$ and the higher gait speed and temporo-spatial parameters when using an ankle-foot orthosis reported earlier. ${ }^{2}$ Further research, particularly assessing the effects of an ankle-foot orthosis on the kinetics at different joints is needed to further test this hypothesis.

The finding that an ankle-foot orthosis can promote dorsiflexion and forward weight transfer during 
stance phase has important clinical implications. At least 90 degrees of dorsiflexion is thought essential for efficient walking, negotiating stairs and kerbs and sitting-down and standing-up, therefore even small improvements could be functionally important and clinically relevant. Our finding of increased dorsiflexion during stance phase is contrary to the common clinical belief that an ankle-foot orthosis detrimentally restricts ankle range of movement during stance, which is often cited as a reason to avoid prescribing an ankle-foot orthosis. Our results indicate that an ankle-foot orthosis could be prescribed to promote dorsiflexion and stability in stance as well as dorsiflexion and heel strike in swing phase.

The review included all designs of ankle-foot orthosis, our aim being to establish the evidence that an ankle-foot orthosis can impact on gait biomechanics rather than to compare designs, and the tested orthoses were very variable in terms of material, shaft design, movement restriction at ankle and footplate length. Further research is needed to compare different designs, define optimal designs and establish algorithms to effectively select the optimal design of ankle foot orthosis for patients with different levels of impairment. An initial step to achieve this would be to develop a standardized tool to classify and describe ankle-foot orthoses, including the most prominent features such as the material properties, flexibility, sole length, shaft height, neck design and weight in all directions.

This review has cautiously shown that an ankle-foot orthosis can improve the biomechanics of gait and offers a mechanism for the improvements in balance and mobility reported earlier. ${ }^{2}$ However many clinicians and patients decline to prescribe, or use an ankle-foot orthosis as many users complain about the weight, discomfort, difficulties fitting it into shoes, or the appearance. ${ }^{32}$ There are also fears that reliance on an ankle-foot orthosis may induce muscle disuse and delay functional recovery. ${ }^{3,33}$ Further research is underway to evaluate short- and long-term effects, adverse effects, adherence and patient satisfaction with different types of ankle-foot orthosis to address these issues.

\section{Clinical messages}

- The available evidence cautiously suggests that an ankle-foot orthosis can reduce energy cost, enhance weight transfer over the weak leg and improve ankle and knee kinematics in people with stroke.

- There was no effect on hip kinematics but these evaluations were probably underpowered.

- There were insufficient common data to analyse the effect on ankle, hip and knee kinetics, muscle activity or spasticity.

\section{Contributions}

SFT initiated and designed the study; screened, extracted and analysed the data; monitored progress and analysis and wrote the paper; ESD contributed to the design, screened, extracted and analysed the data; drafted the paper; CJN contributed to the design, monitored progress; contributed to the paper. SFT acts as guarantor.

\section{Conflict of interest}

The authors declare that there is no conflict of interest.

\section{Funding}

This research received no specific grant from any funding agency, but ESD's PhD (of which this work forms part) was funded by an Overseas Research Scholarship Award from the University of Salford, UK.

\section{References}

1. Bohannon R, Andrews A and Smith M. Rehabilitation goals of patients with hemiplegia. Int J Rehabil Res 1988; 11: 4.

2. Tyson SF and Kent RM. A systematic review and metaanlysis of the effects of an ankle foot orthosis on walking and balance after stroke. Arch Phys Med Rehabil 2013; doi: 10.1016/j.apmr.2012.12.025

3. Leung $J$ and Moseley A. Impact of ankle-foot orthoses on gait and leg muscle activity in adults with hemiplegia: systematic literature review. Physiotherapy 2003; 89: 39-55.

4. PEDro. Physiotherapy Evidence Database, 1999. http:// www.pedro.org.au/english/downloads/pedro-scale/ (accessed 22 February 2011).

5. Higgins J and Thompson S. Quantifying heterogeneity in a meta-analysis. Stat Med 2002; 21: 1539-1558. 
6. Elbourne DR, Altman DG, Higgins JP, Curtin F, Worthington $\mathrm{HV}$ and Vail A. Meta-analyses involving cross-over trials: methodological issues. Int $J$ Epidemiol 2002; 31: 140-149.

7. Hesse S, Luecke D, Jahnke M and Mauritz K. Gait function in spastic hemiparetic patients walking barefoot, with firm shoes, and with ankle-foot orthosis. Int J Rehabil Res 1996; 19: $133-141$

8. Lehmann J, Condon S, Price R and deLateur B. Gait abnormalities in hemiplegia: their correction by ankle-foot orthoses. Arch Phys Med Rehabil 1987; 68: 763-771.

9. Corcoran P, Jebsen R, Brengelmann G and Simons B. Effects of plastic and metal leg braces on speed and energy cost of hemiparetic ambulation. Arch Phys Med Rehabil 1970; 51: 69-77.

10. Pohl M and Mehrholz J. Immediate effects of an individually designed functional ankle-foot orthosis on stance and gait in hemiparetic patients. Clin Rehabil 2006; 20: 324-330.

11. Ibuki A, Bach T, Rogers D and Bernhardt J. The effect of tone-reducing orthotic devices on soleus muscle reflex excitability while standing in patients with spasticity following stroke. Prosthet Orthot Int 2010; 34: 46-57.

12. Burdett R, Borello-France D, Blatchly C and Potter C. Gait comparison of subjects with hemiplegia walking unbraced, with ankle-foot orthosis, and with Air-Stirrup brace. Phys Ther 1988; 68: 1197-1203.

13. Hesse S, Werner C, Matthias K, Stephen K and Berteanu M. Non-velocity-related effects of a rigid double-stopped ankle-foot orthosis on gait and lower limb muscle activity of hemiparetic subjects with an equinovarus deformity. Stroke 1999; 30: 1855-1861.

14. Bleyenheuft C, Caty G, Lejeune T and Detrembleur C. Assessment of the Chignon dynamic ankle-foot orthosis using instrumented gait analysis in hemiparetic adults. Ann Readapt Med Phys 2008; 51: 147-153.

15. Franceschini M, Massucci M, Ferrari L, Agosti M and Paroli C. Effects of an ankle-foot orthosis on spatiotemporal parameters and energy cost of hemiparetic gait. Clin Rehabil 2003; 17: 368-372.

16. Danielsson A and Sunnerhagen K. Energy expenditure in stroke subjects walking with a carbon composite ankle foot orthosis. J Rehabil Med 2004; 36: 165-168.

17. Fatone $\mathrm{S}$ and Hansen A. Effect of ankle-foot orthosis on roll-over shape in adults with hemiplegia. $J$ Rehabil Res Dev 2007; 44: 11-20.

18. Fatone S, Gard S and Malas B. Effect of ankle-foot orthosis alignment and foot-plate length on the gait of adults with poststroke hemiplegia. Arch Phys Med Rehabil 2009; 90: 810-818.

19. Park J, Chun M, Ahn J, Yu J and Kang S. Comparison of gait analysis between anterior and posterior ankle foot orthosisin hemiplegic patients. Am J Phys Med Rehabil 2009; 88: 630-634.
20. Chen C-C, Hong W-H, Wang C-M, et al. Kinematic features of rear-foot motion using anterior and posterior anklefoot orthoses in stroke patients with hemiplegic gait. Arch Phys Med Rehabil 2010; 91: 1862-1868.

21. Erel S, Uygur F, Simsek I and Yakut Y. The effects of dynamic ankle-foot orthoses in chronic stroke patients at three-month follow-up: a randomized controlled trial. Clin Rehabil 2011; 25: 515.

22. Maedaa N, Kato J, Azuma Y, et al. Energy expenditure and walking ability in stroke patients: Their improvement by ankle-foot orthoses. Isokinet Exerc Sci 2009; 17: $57-62$.

23. Mulroy SJ, Eberly VJ, Gronely JK, Weiss W and Newsam CJ. Effect of ankle foot orthosis design on walking after stroke: impact of ankle plantar flexion contracture. Prosthet Orthot Int 2010; 34: 277-292.

24. Nolan KJ and Yarossi M. Preservation of the first rocker is related to increases in gait speed in individuals with hemiplegia and ankle foot orthosis. Clin Biomech 2011; 26: 655-660.

25. Nolan KJ and Yarossi M. Weight transfer analysis in adults with hemiplegia using ankle foot orthosis. Prosthet Orthot Int 2011; 35: 45.

26. Kobayashi T, Leung AKL, Akazawa Y and Hutchins SW. Effect of ankle-foot orthoses on the sagittal plane displacement of the center of mass in patients with stroke hemiplegia: a pilot study. Top Stroke Rehabil 2012; 19: 338-344.

27. Lairamore C, Garrison MK, Bandy WA and Zabel RB. Comparison of tibialis anterior muscle electromyography, ankle angle, and velocity when individuals post stroke walk with different orthoses. Prosthet Orthot Int 2011; 35: 402-410.

28. Yamamoto SA, Fuchi MB and Yasui TC. Change of rocker function in the gait of stroke patients using an ankle foot orthosis with an oil damper: Immediate changes and the short-term effects. Prosthet Orthot Int 2011; 35: 350-359.

29. Gatti MAA, Freixes OA, Fernández SAA, et al. Effects of ankle foot orthosis in stiff knee gait in adults with hemiplegia. J Biomech 2012; 45: 2658-2661.

30. Olney S and Richards C. Hemiparetic gait following stroke. Part I: characteristics. Gait Posture 1996; 4: 12.

31. Riley PO, Della Croce U and Kerrigan DC. Propulsive adaptation to changing gait speed. J Biomech 2001; 34: 197-202.

32. Fisher LR and McLellan DL. Questionnaire assessment of patient satisfaction with lower limb orthoses from a district hospital. Prosthet Orthot Int 1989; 13: 29-35.

33. Geboers J, Drost M, Spaans F, Kuipers H and Seelen H. Immediate and long-term effects of ankle-foot orthosis on muscle activity during walking: a randomized study of patients with unilateral foot drop. Arch Phys Med Rehabil 2002; 83: 240-245. 